\title{
Baryon-baryon interaction of strangeness $S=-1$ sector
}

\author{
Hidekatsu Nemura* ${ }^{\dagger}$ \\ Department of Physics, Tohoku University, Sendai, 980-8578, Japan \\ E-mail: nemura.hidekatsu.gbeu.tsukuba.ac.jp \\ for HAL QCD Collaboration

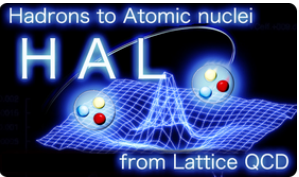

We present our recent studies on hyperon-nucleon $(Y N)$ interactions in the strangeness $S=-1$ that $p \Lambda, \Sigma^{0} p$ and $\Sigma^{+} n$, by extracting corresponding potentials through Nambu-Bethe-Salpeter wave functions. We calculate $\Lambda N$ and $\Sigma N$ potentials in the isospin $I=3 / 2$ channel, using the $N_{f}=$ $2+1$ gauge configurations generated by PACS-CS collaboration and employing an improved method to obtain potentials in lattice QCD simulations. For the ${ }^{1} S_{0}$ channel, the central $\Sigma N(I=$ $\left.3 / 2,{ }^{1} S_{0}\right)$ potential and the central $\Lambda N\left({ }^{1} S_{0}\right)$ potential are found to be very similar. In the spin triplet $\left({ }^{3} S_{1}-{ }^{3} D_{1}\right)$ channels, the central $\Lambda N\left({ }^{3} S_{1}-{ }^{3} D_{1}\right)$ potential is attractive while the central $\Sigma N\left(I=3 / 2,{ }^{3} S_{1}-{ }^{3} D_{1}\right)$ potentials is repulsive. Tensor potentials, on the other hand, are rather weak in the diagonal part of both $\Lambda N$ and $\Sigma N(I=3 / 2)$ systems.

The XXIX International Symposium on Lattice Field Theory - Lattice 2011

July 10-16, 2011

Squaw Valley, Lake Tahoe, California

\footnotetext{
* Speaker.

†Present address: Center of Computational Sciences, University of Tsukuba, Tsukuba, Ibaraki, 305-8577, Japan
} 


\section{Introduction}

The $\Lambda$-nucleon $(\Lambda N)$ and the $\Sigma$-hyperon $(\Sigma N)$ interactions are one of the basic inputs to study the hypernuclear systems. in which hyperons (or strange quarks) are embedded in normal nuclear systems as "impurities" [1]. For example, from studies of few-body systems for $s$-shell $\Lambda$ hypernuclei, it has been pointed out that a coupled-channel $\Lambda N-\Sigma N$ interaction plays a significant role to make a hypernucleus being bounded.[2]. On the other hand, no $\Sigma$-hypernuclei have been observed except for four-body $\Sigma$-hypernucleus $\left({ }_{\Sigma}^{4} \mathrm{He}\right)$, and furthermore a recent experimental study suggests that $\Sigma$-nucleus interaction is repulsive. Such informations are useful to study properties of hyperonic matters inside the neutron stars [3], though a hyperonic equations of state (EOS) employed in such a study may contradict a recent observation of a massive neutron star heavier than $2 M_{\odot}$ [4]. Despite their importance, present phenomenological $\Lambda N$ and $\Sigma N$ interactions have still large uncertainties since direct $\Lambda N$ and $\Sigma N$ scattering experiments are either difficult or impossible due to the short life-time of hyperons.

Recently, a new approach has been proposed lattice QCD to study not only the $N N$ interaction but also various baryonic interactions including the H-dibaryon system [5, 6, 7, 8, , , 10, 11], and the method is recently extended to systems in inelastic channels [12, 13]. This method has been applied also to study various interactions other than baryon-baryon interactions [14, 15]. In this approach, the interparticle potential can be directly extracted in lattice QCD through the NambuBethe-Salpeter (NBS) wave function, and observables such as the phase shift and the binding energy can be calculated by using the resultant potentials. The purpose of this report is to present our recent calculations of the $\Lambda N$ potentials as well as the $\Sigma N(I=3 / 2)$ potentials using full QCD gauge configurations. Several earlier results had already been reported at LATTICE 2008[16] and LATTICE 2009[17]. This report is the latest version of those reports, which includes several new results: (i) $\Sigma N(I=3 / 2)$ potentials are studied. (ii) An improved method is employed to extract potentials more precisely [18].

\section{Improved extraction of potentials}

In the HAL QCD scheme, the non-local but energy-independent potential is defined by the Schrödinger equation as

$$
\left(\vec{\nabla}^{2}+k^{2}\right) \phi(\vec{r})=2 \mu \int d^{3} r^{\prime} U\left(\vec{r}, \vec{r}^{\prime}\right) \phi\left(\vec{r}^{\prime}\right),
$$

where $\phi(\vec{r})$ is the equal-time NBS wave function of two-baryon system $\left(B_{1}, B_{2}\right), \mu=m_{B_{1}} m_{B_{2}} /\left(m_{B_{1}}+\right.$ $\left.m_{B_{2}}\right)$ and $k^{2}$ are the reduced mass of the $\left(B_{1}, B_{2}\right)$ system and the square of asymptotic momentum in the center-of-mass frame, respectively. In practice, the nonlocal potential is expanded in terms of the velocity(derivative) as [19], $U\left(\vec{r}, \vec{r}^{\prime}\right)=V_{B_{1} B_{2}}(\vec{r}, \vec{\nabla}) \delta\left(\vec{r}-\vec{r}^{\prime}\right)$. The potential $V$ may have an antisymmetric spin-orbit term when two baryons are not identical. For example, the $\Lambda N$ potential is given by

$$
V_{\Lambda N}=V_{0}(r)+V_{\sigma}(r)\left(\vec{\sigma}_{\Lambda} \cdot \vec{\sigma}_{N}\right)+V_{T}(r) S_{12}+V_{L S}(r)\left(\vec{L} \cdot \vec{S}_{+}\right)+V_{A L S}(r)\left(\vec{L} \cdot \vec{S}_{-}\right)+O\left(\nabla^{2}\right),
$$

where $S_{12}=3\left(\vec{\sigma}_{\Lambda} \cdot \vec{n}\right)\left(\vec{\sigma}_{N} \cdot \vec{n}\right)-\vec{\sigma}_{\Lambda} \cdot \vec{\sigma}_{N}$ is the tensor operator with $\vec{n}=\vec{r} /|\vec{r}|, \vec{S}_{ \pm}=\left(\vec{\sigma}_{N} \pm \vec{\sigma}_{\Lambda}\right) / 2$ are symmetric (+) and antisymmetric (-) spin operators, $\vec{L}=-i \vec{r} \times \vec{\nabla}$ is the orbital angular momentum 
operator. In the velocity expansion, $V_{0, \sigma, T}$ are the leading order (LO) potentials, denoted by $V_{\mathrm{LO}}$, while $V_{L S, A L S}$ are of the next-to-leading-order (NLO). In this report, we consider the LO potentials only.

In lattice QCD simulations we first calculate the normalized four-point correlation function defined by

$$
R_{\alpha \beta}^{(J, M)}\left(\vec{r}, t-t_{0}\right)=\sum_{\vec{X}}\left\langle 0\left|B_{1, \alpha}(\vec{X}+\vec{r}, t) B_{2, \beta}(\vec{X}, t) \overline{\mathscr{J}_{B_{3} B_{4}}^{(J, M)}\left(t_{0}\right)}\right| 0\right\rangle / \exp \left\{-\left(m_{B_{1}}+m_{B_{2}}\right)\left(t-t_{0}\right)\right\},
$$

where the summation over $\vec{X}$ selects states with zero total momentum. The $B_{1, \alpha}(x)$ and $B_{2, \beta}(y)$ denote the interpolating fields of the baryons such as

$$
\begin{aligned}
& p=\varepsilon_{a b c}\left(u_{a} C \gamma_{5} d_{b}\right) u_{c}, \quad n=-\varepsilon_{a b c}\left(u_{a} C \gamma_{5} d_{b}\right) d_{c}, \\
& \Sigma^{+}=-\varepsilon_{a b c}\left(u_{a} C \gamma_{5} s_{b}\right) u_{c}, \quad \Sigma^{0}=\frac{1}{\sqrt{2}} \varepsilon_{a b c}\left\{\left(d_{a} C \gamma_{5} s_{b}\right) u_{c}+\left(u_{a} C \gamma_{5} s_{b}\right) d_{c}\right\} \\
& \Lambda=\frac{1}{\sqrt{6}} \varepsilon_{a b c}\left\{\left(d_{a} C \gamma_{5} s_{b}\right) u_{c}+\left(s_{a} C \gamma_{5} u_{b}\right) d_{c}-2\left(u_{a} C \gamma_{5} d_{b}\right) s_{c}\right\}
\end{aligned}
$$

and $\overline{\mathscr{J}_{B_{3} B_{4}}^{(J, M)}\left(t_{0}\right)}=\sum_{\alpha^{\prime} \beta^{\prime}} P_{\alpha^{\prime} \beta^{\prime}}^{(J, M)} \overline{B_{3, \alpha^{\prime}}\left(t_{0}\right) B_{4, \beta^{\prime}}\left(t_{0}\right)}$ is a source operator which creates $B_{3} B_{4}$ states with the total angular momentum $J, M$. This normalized four-point function can be expressed as

$$
R_{\alpha \beta}^{(J, M)}\left(\vec{r}, t-t_{0}\right)=\sum_{n} A_{n} \sum_{\vec{X}}\left\langle 0\left|B_{1, \alpha}(\vec{X}+\vec{r}, t) B_{2, \beta}(\vec{X}, t)\right| E_{n}\right\rangle \mathrm{e}^{-\left(E_{n}-m_{B_{1}}-m_{B_{2}}\right)\left(t-t_{0}\right)},
$$

where $E_{n}\left(\left|E_{n}\right\rangle\right)$ is the eigen-energy (eigen-state) of the six-quark system with the particular quantum number (i.e., $J^{\pi}, M$, strangeness $S$ and isospin $I$ ), and $A_{n}=\sum_{\alpha^{\prime} \beta^{\prime}} P_{\alpha^{\prime} \beta^{\prime}}^{(J M)}\left\langle E_{n}\left|\bar{B}_{4, \beta^{\prime}} \bar{B}_{3, \alpha^{\prime}}\right| 0\right\rangle$.

Since $E_{n}-m_{B_{1}}-m_{B_{2}}=k^{2} /(2 \mu)+O\left(k^{4}\right)$, we have [18]

$$
\left(\frac{\nabla^{2}}{2 \mu}-\frac{\partial}{\partial t}\right) R(\vec{r}, t)=\int d^{3} r^{\prime} U\left(\vec{r}, \vec{r}^{\prime}\right) R\left(\vec{r}^{\prime}, t\right)+O\left(k^{4}\right)=V_{\mathrm{LO}}(\vec{r}) R(\vec{r}, t)+\cdots,
$$

where $t-t_{0}$ should be moderately large so that states with large $k^{2}$ and states with more than 2 particles are suppressed.

For the spin singlet state, we extract the central potential as $V_{C}(r ; J=0)=\left(\frac{\nabla^{2}}{2 \mu}-\frac{\partial}{\partial t}\right) R / R$. For the spin triplet state, the wave function is decomposed into the $S$ - and the $D$-wave components as

$$
\left\{\begin{array}{l}
R_{\alpha \beta}\left(\vec{r} ;{ }^{3} S_{1}\right)=\mathscr{P} R_{\alpha \beta}(\vec{r} ; J=1) \equiv \frac{1}{24} \sum_{\mathscr{R} \in O} \mathscr{R} R_{\alpha \beta}(\vec{r} ; J=1) \\
R_{\alpha \beta}\left(\vec{r} ;{ }^{3} D_{1}\right)=\mathscr{Q} R_{\alpha \beta}(\vec{r} ; J=1) \equiv(1-\mathscr{P}) R_{\alpha \beta}(\vec{r} ; J=1) .
\end{array}\right.
$$

Therefore, the Schrödinger equation with the LO potentials for the spin triplet state becomes

$$
\left\{\begin{array}{l}
\mathscr{P} \\
\mathscr{Q}
\end{array}\right\} \times\left\{-\frac{\nabla^{2}}{2 \mu}+V_{0}(r)+V_{\sigma}(r)\left(\vec{\sigma}_{\Lambda} \cdot \vec{\sigma}_{N}\right)+V_{T}(r) S_{12}\right\} R\left(\vec{r}, t-t_{0}\right)=-\left\{\begin{array}{l}
\mathscr{P} \\
\mathscr{Q}
\end{array}\right\} \times \frac{\partial}{\partial t} R\left(\vec{r}, t-t_{0}\right),
$$

from which the central and the tensor potentials, $V_{C}(r ; J=0)=V_{0}(r)-3 V_{\sigma}(r)$ for $J=0, V_{C}(r ; J=$ $1)=V_{0}(r)+V_{\sigma}(r)$, and $V_{T}(r)$ for $J=1$, can be determined. 

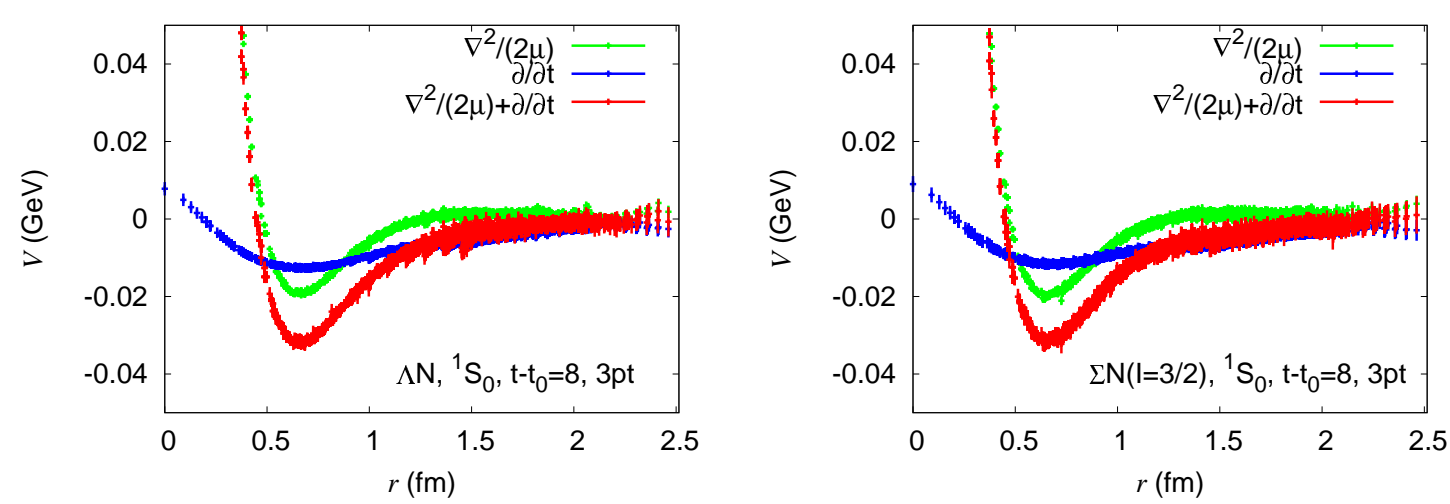

Figure 1: Left: The central potential (red) in the ${ }^{1} S_{0}$ channel of the $\Lambda N$ system in $2+1$ flavor QCD as a function of $r$, together with the $\frac{\nabla^{2}}{2 \mu}$ part (green), $\frac{\partial}{\partial t}$ part (blue). Right: The central potential in the ${ }^{1} S_{0}$ channel of the $\Sigma N(I=3 / 2)$ system as a function of $r$. Symbols are same as the left figure.

\section{Numerical simulations}

In our calculations for $\Lambda N$ and $\Sigma N(I=3 / 2)$ potentials, we employ $2+1$ flavor full QCD gauge configurations generated by PACS-CS collaboration [23] with the RG-improved Iwasaki gauge action and the nonperturbatively $O(a)$-improved Wilson quark action at $\beta=1.9$ on a $L^{3} \times T=$ $32^{3} \times 64$ lattice. The lattice spacing at the physical quark masses is $a=0.0907(13) \mathrm{fm}$ [23], thus the spatial volume is $(2.90 \mathrm{fm})^{3}$. We have chosen one set of hopping parameters $\left(\kappa_{u d}, \kappa_{s}\right)=$ $(0.13700,0.13640)$ for light and the strange quarks, which correspond to $\left(m_{\pi}, m_{K}\right) \cong(699,787)$ $\mathrm{MeV}$. The wall source with the Coulomb gauge fixing is placed at the time-slice $t_{0}$, and the Dirichlet boundary condition is imposed in the temporal direction at the time-slice $t-t_{0}=N_{t} / 2$. A total number of gauge configurations we have used is 399 , and we put a source at $t_{0}=8 n$ with $n=$ $0,1,2, \cdots, 8$ on each configuration to increase the statistics.

\section{Results}

\section{1 $\Lambda N$ and $\Sigma N(I=3 / 2)$ potentials in ${ }^{1} S_{0}$ channel}

The $\Lambda N$ (left panel) and the $\Sigma N(I=3 / 2)$ (right panel) potentials in the ${ }^{1} S_{0}$ channel are shown in Figure 1 where the laplacian part is represented by green, the time-derivative part by blue and the total potential by red, respectively. Note that the time derivative term gives a significant attraction at medium and longer distances.

In the $2+1$ flavor QCD, while the $\Sigma N(I=3 / 2)$ potential still belongs directly to the $27(I=$ $3 / 2$ ) representation thanks to the isospin $(I)$ symmetry, an energy eigenstate of a $\Lambda N$ system in the ${ }^{1} S_{0}$ channel is a mixture of $\mathbf{2 7}(I=1 / 2)$ and $\mathbf{8}_{s}$ in the flavor representation, so that these two potentials are not necessarily mutually in agreement. As seen from Figure 1, however, these two potentials look very similar to each other, since the flavor symmetry breaking is still small in the present $2+1$ flavor QCD calculation, where the baryon masses are given by $\left(m_{N}, m_{\Lambda}, m_{\Sigma}\right)=$ (1.574(3), 1.635(3), 1.650(3)) GeV. 

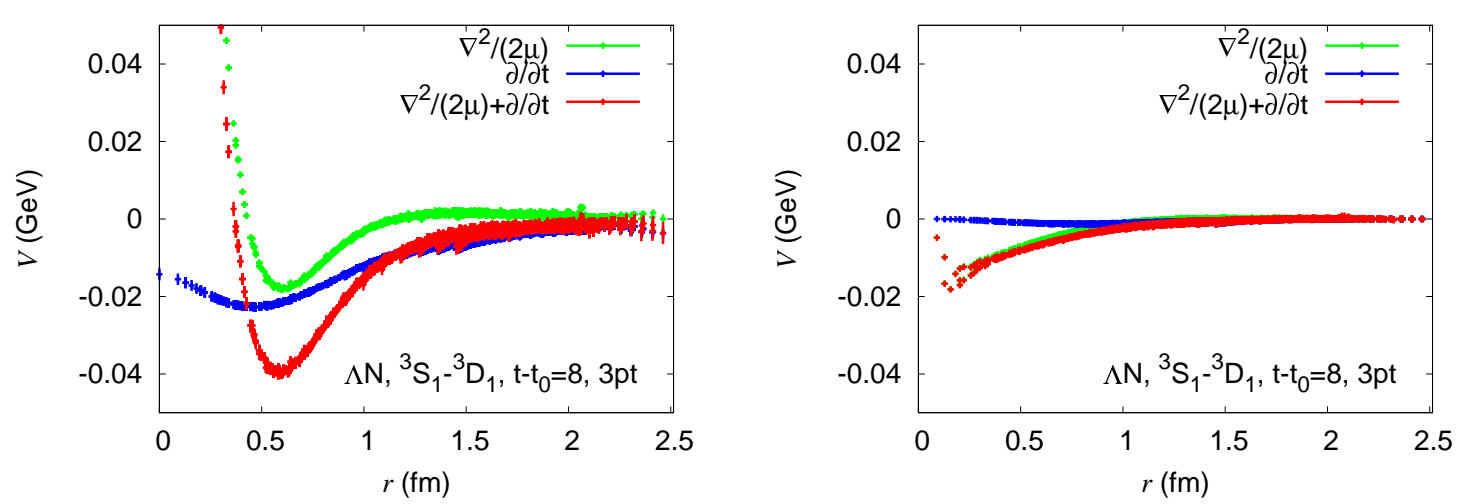

Figure 2: Left: The central potential in the ${ }^{3} S_{1}-{ }^{3} D_{1}$ channel of the $\Lambda N$ system as a function of $r$. Symbols are same as the Fig. 1 Right: The tensor potential in the ${ }^{3} S_{1}-{ }^{3} D_{1}$ channel of the $\Lambda N$ system as a function of $r$. Symbols are same as Fig. 1.

\section{2 $\Lambda N$ interaction in the ${ }^{3} S_{1}-{ }^{3} D_{1}$ channel}

Figure2 2 shows the central potential (left panel) and the tensor potential (right panel) of the $\Lambda N$ system in the ${ }^{3} S_{1}-{ }^{3} D_{1}$ channel, whose eigenstate is a mixture of $\overline{\mathbf{1 0}}$ and $\mathbf{8}_{a}$. The time-derivative part (blue) of the central potential has attractive contributions, so that the attraction of the total potential (red) is enhanced at long distance. The attractive well at the distance $r \approx 0.6 \mathrm{fm}$ is deeper than that of the $\Lambda N$ central potential in the ${ }^{1} S_{0}$ channel. The time derivative part of the tensor potential, on the other hand, gives a negligible contribution. Furthermore, the tensor potential itself (red) is weaker than the tensor potential of the $N N$ system [18].

\section{3 $\Sigma N$ interaction in the ${ }^{3} S_{1}-{ }^{3} D_{1}$ channel}

Figure 3 shows the central potential (left panel) and the tensor potential (right panel) of the $\Sigma N(I=3 / 2)$ system in the ${ }^{3} S_{1}-{ }^{3} D_{1}$ channel. Due to the isospin symmetry, this channel belongs solely to the flavor $\mathbf{1 0}$ representation without mixing with $\overline{\mathbf{1 0}}$ or $\mathbf{8}_{a}$

As seen form an enlargement in the inset of the left panel, there is no clear attractive well in the central potential (red). An attractive well seen in the laplacian part (green) at medium distances is cancelled by a repulsive contribution in the time-derivative part (blue) at the whole range. This repulsive nature of the $\Sigma N\left(I=3 / 2,{ }^{3} S_{1}-{ }^{3} D_{1}\right)$ central potential is consistent with the prediction from the naive quark model. 24] The tensor force is a little stronger that that of the $\Lambda N$ system but is still weaker in magnitude than that of the $N N$ system. The time-derivative part gives a negligible contribution.

\section{Summary}

We have calculated the central and the tensor parts of the $\Lambda N$ and $\Sigma N(I=3 / 2)$ potentials using the improved method. Two potentials in the ${ }^{1} S_{0}$ channel, one is $\Lambda N$ and another is $\Sigma N(I=3 / 2)$, are very similar to each other, since both sectors have a common 27 representation in the flavor $\mathrm{SU}(3)$ and the SU(3) breaking effect is still small in the present calculation. Both potentials have an attractive well, so that they give attractive interactions at low energy. 

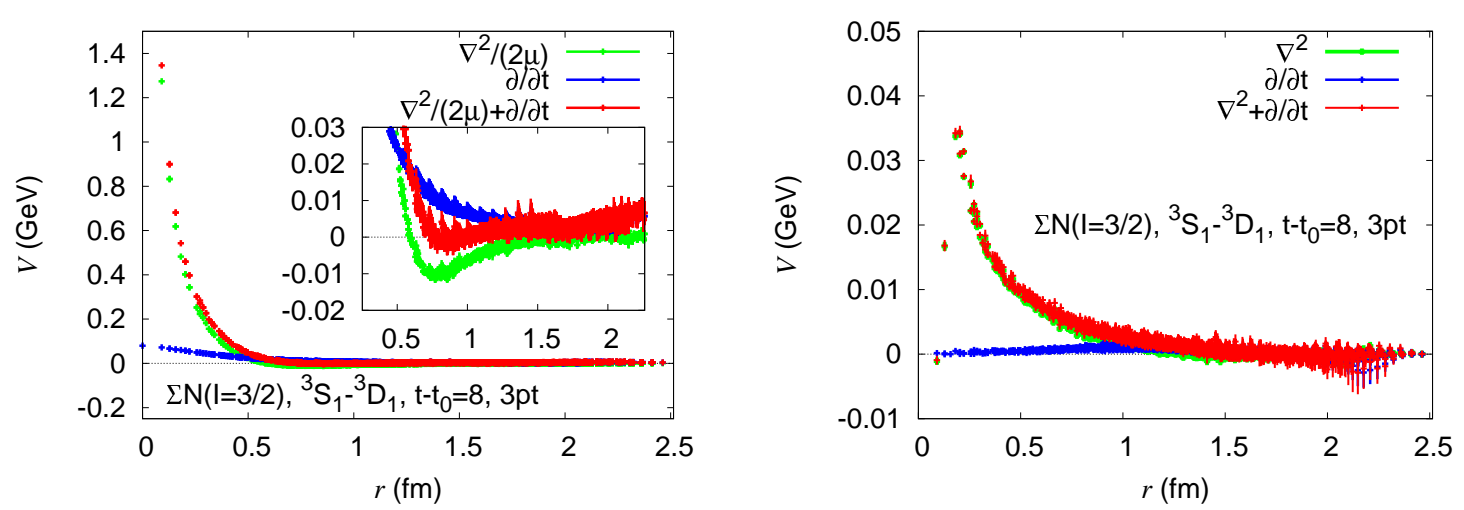

Figure 3: Left: The central potential in the ${ }^{3} S_{1}-{ }^{3} D_{1}$ channel of the $\Sigma N$ system as a function of $r$. Right: The tensor potential in the ${ }^{3} S_{1}-{ }^{3} D_{1}$ channel of the $\Sigma N$ system as a function of $r$.

In the ${ }^{3} S_{1}-{ }^{3} D_{1}$ channel, while the central $\Lambda N$ potential shows attraction at low energy with an attractive well, the central $\Sigma N(I=3 / 2)$ potential is repulsive at all distances. Tensor potentials for both $\Lambda N$ and $\Sigma N(I=3 / 2)$ systems in this work are rather weak.

In this report, we have extracted single-channel potentials only without coupled-channel analysis between $\Lambda N$ and $\Sigma N$. though the coupled-channel potentials can be also extracted in principle 12 [13]. The author had postponed such a coupled-channel analysis to future publications, since he had to spend his time to recover in various parts of his life and research environment from the massive earthquake in East of Japan on March 11th, 2011. Further analyses including coupled-channel potentials are now in progress.

\section{Acknowledgments}

The author would like to thank CP-PACS/JLQCD collaborations, PACS-CS Collaboration and ILDG/JLDG 21] for allowing us to access the full QCD gauge configurations, and Dr. T. Izubuchi for providing a sample FFT code. The author also thank maintainers of CP S++[25]. Calculations in this report have been performed by using the Blue Gene/L computer under the "Large scale simulation program" at KEK (No. 10-24). He would also like to thank Dr. S. Ejiri, Dr. K. Itahashi and Advanced Meson Science Laboratory of RIKEN Nishina Center for providing a special computer resource, and Prof. H. Tamura and Dr. T. Koike for generous support in recovering the research environment after the massive earthquake in East of Japan on Math 11th. The author is supported by the Global COE Program for Young Researchers at Tohoku University. This research was supported in part by Strategic Program for Innovative Research (SPIRE), the MEXT Grant-in-Aid, Scientific Research on Innovative Areas (Nos. 21105515, 20105003).

\section{References}

[1] Reviewed in O. Hashimoto and H. Tamura, Prog. Part. Nucl. Phys. 57, 564 (2006).

[2] H. Nemura, Y. Akaishi and Y. Suzuki, Phys. Rev. Lett. 89, 142504 (2002) [arXiv:nucl-th/0203013].

[3] See e.g. J. Schaffner-Bielich, Nucl. Phys. A 804, 309 (2008) [arXiv:0801.3791 [astro-ph]]. 
[4] P. B. Demorest, et al., Nature 467, 1081 (2010).

[5] N. Ishii, S. Aoki, T. Hatsuda, Phys. Rev. Lett. 99, 022001 (2007).

[6] S. Aoki, T. Hatsuda and N. Ishii, Prog. Theor. Phys. 123 (2010) 89.

[7] K. Murano, N. Ishii, S. Aoki and T. Hatsuda, Prog. Theor. Phys. 125 (2011) 1225.

[8] H.Nemura, N.Ishii, S.Aoki and T.Hatsuda, Phys. Lett. B 673, 136 (2009).

[9] T. Inoue et al. [HAL QCD collaboration], Prog. Theor. Phys. 124 (2010) 591.

[10] T. Inoue et al. [HAL QCD Collaboration], Phys. Rev. Lett. 106 (2011) 162002.

[11] T. Doi et al., [HAL QCD Collaboration], arXiv:1106.2276 [hep-lat].

[12] S. Aoki et al. [HAL QCD Collaboration], Proc. Jpn. Acad. B 87, 509 (2011) [arXiv:1106.2281 [hep-lat]].

[13] K. Sasaki for HAL QCD Collaboration, in these proceedings.

[14] Y. Ikeda [for HAL QCD Collaboration], arXiv:1111.2663 [hep-lat].

[15] Y. Ikeda and H. Iida, arXiv:1102.2097 [hep-lat].

[16] H. Nemura, N. Ishii, S. Aoki and T. Hatsuda [PACS-CS Collaboration], PoS LATTICE2008, 156 (2008) [arXiv:0902.1251 [hep-lat]].

[17] H. Nemura [HAL QCD Collaboration and PACS-CS Collaboration], PoS LAT2009, 152 (2009) [arXiv:1005.5352 [hep-lat]].

[18] N. Ishii for HAL QCD collaboration, in these proceedings.

[19] R. Tamagaki and W. Watari, Prog. Theor. Phys. Suppl. 39, 23 (1967).

[20] S. Okubo and R.E. Marshak, Ann. Phys. (NY) 4, 166 (1958).

[21] See http://www. lqcd.org/ildg and http://www.jldg.org

[22] T. Ishikawa et al. [CP-PACS/JLQCD Collaboration], Phys. Rev. D 78 (2008) 011502(R).

[23] S. Aoki et al. [PACS-CS Collaboration], Phys.Rev.D79, 034503 (2009) [arXiv:0807.1661 [hep-lat]].

[24] M. Oka, K. Shimizu and K. Yazaki, Prog. Theor. Phys. Suppl. 137, 1 (2000).

[25] Columbia Physics System (CPS), http: / / qcdoc.phys. columbia . edu/cps.html. 